\section{Características sociodemográficas e de assistência à gestação e ao parto no extremo sul do Brasil}

\author{
Socio-demographic characteristics and prenatal \\ and childbirth care in southern Brazil
}

\author{
1 Divisão de População \& \\ Saúde, Universidade Federal \\ do Rio Grande, Rio Grande, \\ Brasil. \\ 2 Programa de Pós-graduação \\ em Epidemiologia, \\ Universidade Federal de \\ Pelotas, Pelotas, Brasil. \\ 3 Mestrado em Ciências da \\ Saúde, Universidade Federal \\ do Rio Grande, Rio Grande, \\ Brasil. \\ 4 Faculdade de Medicina \\ Universidade Federal do Rio \\ Grande, Rio Grande, Brasil. \\ Correspondência \\ J. A. Cesar \\ Divisão de População \& \\ Saúde, Universidade Federal \\ do Rio Grande. \\ Rua Lisboa 1156, Pelotas, RS \\ 96083-210, Brasil. \\ jacesar@terra.com.br
}

\section{Abstract}

This study aimed to compare prenatal and childbirth care received by teenagers and older mothers in Rio Grande, Rio Grande do Sul State, southern Brazil. From January 1st to December 31st 2007, all mothers were interviewed with a standardized questionnaire on the care they received. The chi-square test was used to compare proportions between adolescent and non-adolescent mothers. One-fourth (516) of the infants were born to adolescent mothers. Compared to older mothers, teenagers showed lower rates of the following: completion of at least six prenatal visits $(61 \% x$ $75 \%)$, initiation of prenatal care in the first trimester (58\% $x$ 77\%), tetanus vaccination ( $81 \% x$ $85 \%)$, and completion of prenatal visits with the same health professional (70\% $x$ 78\%). Meanwhile, teenage motherhood was associated with more: supplementation for iron deficiency (66\% $x 57 \%$ ), use of forceps (11\% x 6\%), and episioto$m y$ (86\% $x$ 66\%). The findings show that teenage mothers received worse prenatal and childbirth care than older mothers.

Maternal and Child Health; Prenatal Care; Pregnancy; Adolescent
Juraci A. Cesar 1,2

Raúl A. Mendoza-Sassi 1

David A. Gonzalez-Chica 2

Patrícia S. Mano ${ }^{3}$

Sirley M. Goulart-Filha 4

\section{Introdução}

A assistência pré-natal tem por objetivo reduzir a morbimortalidade materno-fetal. Esta redução depende tanto dos cuidados recebidos no período gestacional quanto da assistência recebida no momento do parto. Exames clínicos e laboratoriais oferecidos durante as consultas de pré-natal permitem identificar situações de risco e agir precocemente, enquanto a assistência recebida no momento do parto é o principal determinante da morbimortalidade no período neonatal 1,2,3.

Uma das situações que implica maior risco à mortalidade infantil é o fato de a gestação ocorrer entre adolescentes, ou seja, entre aquelas com menos de 20 anos de idade. A adolescência esta relacionada à maior ocorrência de prematuridade e baixo peso ao nascer e pré-natal inadequado, que implica menor número de consultas de pré-natal, início das consultas mais tardiamente e baixa realização de exames complementares 2,3,4,5. Somam-se a estes fatores a falta de informações sobre os benefícios do pré-natal e o sentimento de vergonha pela busca de cuidados médicos por parte das adolescentes, sobretudo entre as mais pobres 6 . Além disso, elas amamentam seus filhos por um menor período de tempo 7 . Isto tudo resulta em maior mortalidade infantil entre seus filhos 1,3.

A ocorrência de gravidez na adolescência não é um achado raro no Brasil. Uma em cada cinco brasileiras tem o primeiro filho antes dos 20 anos 
de idade. E esta proporção tem se mantido constante nos últimos 10 anos, apesar de queda acentuada na taxa de fecundidade no mesmo período da ordem de $12 \%$, em média, em todo o país. No Rio Grande do Sul, houve ligeira redução na taxa de gravidez nesta mesma faixa etária, passando de $20,2 \%$ em 1999 para 17,4\% em 2008, o que representou 50 mil nascimentos a menos no estado neste período. Por fim, no Município de Rio Grande, local em que este estudo foi conduzido, a taxa de gravidez caiu de 22,5\% em 1999 para 20,2\% em 2008, ou 1.100 nascimentos a menos no período (Departamento de Informática do SUS. http://tabnet.datasus.gov.br/cgi/idb2008/ a05a.htm, acessado em 19/Jun/2010).

Um evento que afeta uma quarto da população e que se mostra associado à ocorrência de doença e morte é um problema de saúde pública que merece investigação. Além disso, são poucos os estudos que comparam a qualidade da assistência recebida durante a gestação e o parto entre adolescentes em relação às demais gestantes. É disto que trata este artigo que incluiu todas as gestantes que deram à luz no Município de Rio Grande, em 2007.

\section{Métodos}

Foram incluídos neste estudo todos os nascimentos ocorridos nas duas únicas maternidades do Município de Rio Grande, extremo sul do Brasil. As maternidades foram da Santa Casa de Misericórdia e do Hospital Universitário da Universidade Federal do Rio Grande. Entre 1o de janeiro e 31 de dezembro de 2007, todas as mães residentes no município, cujo filho tenha nascido com peso igual ou superior a $500 \mathrm{~g}$ e/ou pelo menos 20 semanas de idade gestacional, foram entrevistadas.

Utilizou-se delineamento transversal (seccional ou de prevalência) 8 com as mães sendo entrevistadas na maternidade, a grande maioria delas nas primeiras 24 horas após o parto.

Foram treinados 10 entrevistadores, sendo oito deles acadêmicos do Curso de Medicina da Universidade Federal do Rio Grande (FURG) e as outras duas graduadas em serviço social e com ampla experiência na aplicação de entrevistas em nível hospitalar. Ao longo de quatro dias de treinamento, realizou-se leitura do questionário e do manual de instruções, aplicação entre duplas e perante o grupo de entrevistadores. $\mathrm{O}$ estudo piloto foi realizado nas mesmas maternidades cerca de duas semanas antes do início da coleta de dados. Nesta etapa, cada um dos treinandos aplicou pelo menos dois questionários completos. Em seguida, foram sanadas as últimas dúvidas e realizadas as alterações no questionário.

Estes entrevistadores aplicaram questionário único pré-codificado, com perguntas fechadas buscando informações sobre características demográficas (idade, cor da pele e estado civil), história reprodutiva materna (número de filhos tidos vivos e mortos, ocorrência prévia de abortos e idade por ocasião do primeiro parto); nível socioeconômico (escolaridade e renda familiar); assistência recebida durante a gestação (idade de inicio do pré-natal, número de consultas, imunização antitetânica, local de realização do prénatal, se realizou todo o pré-natal com o mesmo profissional, tipo de exames clínicos e laboratoriais a que foi submetida e se foram atendidas na rede pública ou privada); relato de morbidade no período gestacional (hipertensão, diabetes, anemia, corrimento vaginal e ameaça de aborto e de parto prematuro); atendimento ao parto (profissional que fez o parto, tipo de parto, uso de fórcipe e realização de episiotomia); e características do recém-nascido [sexo, peso e comprimento ao nascer, índice de Apgar e idade gestacional, esta avaliada a partir da data da última menstruação (DUM) anotada no cartão da gestante]. Considerou-se como adolescente toda parturiente com menos de 20 anos de idade.

Foram utilizados dois índices para avaliar a adequação do pré-natal, ambos baseados no proposto por Kessner ${ }^{9}$, que foram os de Takeda 10 e Silveira et al. ${ }^{11}$. Na avaliação proposta por Takeda 10 combina-se o número total de consultas com o momento de ingresso ao pré-natal e três categorias são estabelecidas: adequado, quando são realizadas seis ou mais consultas e cujo início do pré-natal se dá antes de cinco meses de gestação; inadequado, quando são realizadas menos de três consultas de pré-natal ou quando o início destas consultas se dá após o sétimo mês de gestação; e intermediário, que engloba as demais situações. Silveira et al. 11 classificam como adequado a realização de seis ou mais consultas antes do quinto mês de gestação associados à realização de dois ou mais exames qualitativos de urina, hemoglobina e VDRL; inadequado para três consultas, cujo início se dá após o sétimo mês de gestação sem a realização de qualquer um destes exames laboratoriais e, por fim, intermediário para as demais situações. Nas duas avaliações, os dados não lembrados pelas gestantes foram classificados na categoria "ignorado".

Durante o período de coleta de dados, estes entrevistadores visitavam diariamente as duas maternidades da cidade. A identificação das gestantes era feita diretamente com a parturiente e confirmada através do livro de registro de nascimento de cada maternidade. Ao encontrar 
alguma parturiente residente no Município de Rio Grande, um termo de consentimento era por ela assinado permitindo a entrevista. O questionário resultante desta entrevista era codificado ao final de cada dia de trabalho e, no dia seguinte, entregue na sede do estudo onde eram revisados e entregues à digitação.

As entrevistadoras de maior experiência aplicavam o questionário nos dias úteis, enquanto os estudantes, atuando sempre em duplas, ficavam responsáveis pela realização das entrevistas nos finais de semana e durante feriados. A cada mês, realizava-se rodízio a fim de possibilitar que todos atuassem nas duas maternidades em períodos diferentes.

Ao final de cada dia de trabalho, cada entrevistador codificava os questionários e os entregava no dia seguinte na sede do projeto, localizado em um anexo do Hospital Universitário. Quinzenalmente, a equipe se reunia com um dos coordenadores do estudo (J.A.C./R.A.M.S.) a fim de avaliar o andamento do trabalho e fazer as mudanças que se mostrassem necessárias.

Dez por cento das entrevistas foram refeitas a fim de avaliar a qualidade dos dados coletados. Isto foi feito com as mães em suas residências por telefone ou através de visita. Nesta ocasião, aplicava-se um questionário resumido.

Os questionários eram duplamente digitados em ordem inversa por diferentes profissionais. A cada duas semanas, estas digitações eram comparadas e eventuais erros eram listados e corrigidos. Em seguida, verificava-se a consistência dos dados com imediata correção de eventuais diferenças. A entrada de dados foi realizada utilizando-se do programa Epi Info 6.04 (Centers for Disease Control and Prevention, Atlanta, Estados Unidos), enquanto a análise de consistência, que incluiu a categorização de variáveis e verificação de frequências, foi realizada através do pacote estatístico Stata versão 9.2 (Stata Corp., College Station, Estados Unidos). Esta análise consistiu de verificação de frequências e comparação de proporções através do teste do qui-quadrado de Pearson 12.

O protocolo de pesquisa foi submetido e aprovado pelo Comitê de Ética em Pesquisa na Área da Saúde (CEPAS) da Universidade Federal do Rio Grande. Além disso, garantiu-se a confidencialidade dos dados, a participação voluntária e a possibilidade de deixar o estudo a qualquer momento, sem necessidade de justificativa.

\section{Resultados}

Entre 1o de janeiro e 31 de dezembro de 2007 houve 2.584 nascimentos no Município de Rio Grande. Este estudo obteve informações sobre 2.557 deles, o que representa $98,9 \%$ do total. Destes, $2.523(98,7 \%)$ nasceram de partos únicos e $34(1,3 \%)$ de parto gemelar; 39 (1,5\%) nasceram mortos, $33(1,1 \%)$ mães não foram encontradas ou tiveram o parto domiciliar e uma recusou-se a participar do estudo $(\mathrm{p}>0,05)$. Um quinto delas (516) era constituído de adolescentes $(<20$ anos).

A Tabela 1 mostra que mães adolescentes possuíam, em média, três anos a menos de escolaridade em relação às demais mães, e que, enquanto apenas $30 \%$ delas haviam completado o ensino médio, o mesmo ocorria para $45 \%$ daquelas de maior idade. Em relação à renda familiar, verificou-se que a proporção de adolescentes no primeiro quartil de renda, o mais baixo, foi cerca de duas vezes maior em relação às mães de maior idade ( $41 \%$ x 23\%). Por fim, a proporção de mães adolescentes vivendo sem companheiro foi 2,6 vezes maior em relação às demais (35\% x 13\%).

Na Tabela 2, é possível verificar que as adolescentes realizaram um número substancialmente maior de consultas de pré-natal nos postos de saúde, enquanto que a proporção de mães de maior idade que realizou pré-natal com médico particular foi cerca de duas vezes maior. Estas mães de maior idade mostraram ainda vantagem estatisticamente significativa em relação às adolescentes no que diz respeito à realização de seis ou mais consultas de pré-natal (75\% x 61\%), inicio do pré-natal no primeiro trimestre $(77 \%$ x 58\%), realização de pré-natal com o mesmo profissional (78 x 71\%) e realização de ultrassonografia pélvica (93\% x 89\%). Estas diferenças mantiveram-se favoráveis às mães de maior idade em relação ao exame de mamas (47\% x 39\%), realização de citopatológico de colo uterino (37\% x 30\%) e imunização antitetânica (85\% x 81\%). As adolescentes, por sua vez, foram mais comumente suplementadas com sulfato ferroso $(66 \%$ x 57\%) e referiram com maior frequência ameaça de parto prematuro $(63 \% \times 49 \%)$ e corrimento vaginal patológico (52\% x 37\%). Apesar disso, usaram menos medicamentos que as demais mães no período gestacional (71\% x 64\%).

A assistência ao parto é mostrada na Tabela 3. Esta tabela mostra que, em relação às demais mães, as adolescentes foram menos frequentemente atendidas por médicos no pré-parto $(28 \%$ x 25\%), tiveram filhos mais comumente através de parto normal $(60 \%$ x $46 \%)$, foram em maior frequência submetidas ao uso de fórcipe ( $11 \%$ x 6\%) e episiotomia ( $86 \%$ x 66\%) e a assistência 
Características demográficas maternas e sócio-econômicas das famílias de recém-nascidos. Município de Rio Grande, Rio Grande do Sul, Brasil, 2007.

\begin{tabular}{|c|c|c|c|}
\hline Variável & Mães adolescentes & Mães não adolescentes & Valor de $p$ * \\
\hline \multicolumn{4}{|l|}{ Cor da pele da mãe (autorreferida) } \\
\hline Branca & $63,1 \%$ & $67,5 \%$ & 0,2 \\
\hline Parda/Morena & $14,0 \%$ & $13,1 \%$ & \\
\hline Preta & $22,9 \%$ & $19,5 \%$ & \\
\hline \multicolumn{4}{|l|}{ Escolaridade em anos completos } \\
\hline $0-4$ & $8,9 \%$ & $13,5 \%$ & $<0,001$ \\
\hline $5-8$ & $59,7 \%$ & $30,1 \%$ & \\
\hline $9-11$ & $30,2 \%$ & $44,8 \%$ & \\
\hline 12 ou mais & $1,2 \%$ & $11,5 \%$ & \\
\hline Mediana (intervalo interquartil) & $7(6-9)$ & $10(6-11)$ & $<0,001 * \star$ \\
\hline \multicolumn{4}{|l|}{ Renda familiar total (quartis) } \\
\hline Primeiro (inferior) & $41,1 \%$ & $22,9 \%$ & $<0,001$ \\
\hline Segundo & $28,3 \%$ & $25,9 \%$ & \\
\hline Terceiro & $19,0 \%$ & $23,8 \%$ & \\
\hline Quarto (superior) & $11,6 \%$ & $27,4 \%$ & \\
\hline Mediana de renda per capita em $\mathrm{R} \$$ (intervalo interquartil) & $187,5(100-272)$ & $260(133-500)$ & $<0,001 * \star$ \\
\hline Media de moradores por domicílio & 3,9 & 3,6 & $0,4 * \star \star$ \\
\hline Vivem com companheiro & $65,1 \%$ & $87,3 \%$ & $<0,001$ \\
\hline Total & 516 & 2.041 & \\
\hline
\end{tabular}

* Teste de qui-quadrado de Fisher;

** Teste de qui-quadrado Pearson para comparação de valores acima da mediana;

*** Teste de Kruskal-Wallis.

recebida foi através do SUS (92\% x 76\%). Todas estas diferenças foram estatisticamente significativas $(\mathrm{p}<0,05)$.

Na Tabela 4 verifica-se ausência de diferença significativa no que diz respeito a vários desfechos do pós-parto imediato. Por exemplo, entre todas as mães, a prevalência de baixo peso ao nascer ficou ao redor de 7\%; a média de comprimento ao nascer foi de $48 \mathrm{~cm}$ enquanto que para o perímetro cefálico foi de $34 \mathrm{~cm}$. Houve pequena diferença na ocorrência de parto prematuro $(<37$ semanas de gestação). Entre as mães adolescentes foi de $20 \%$ contra $16 \%$ entre as mães de maior idade, mas sem alcançar significância estatística. Vale destacar ainda que para um quarto de todas as mães não foi possível obter informações quanto à idade gestacional em virtude de as mães não se lembrarem da DUM. Finalmente, também para todas elas, a prevalência de natimortos foi de $1,5 \%$ enquanto o índice Apgar $\geq 8$ no quinto minuto foi de $96 \%$ nos dois grupos.

A Figura 1 mostra que em relação à avaliação da qualidade do pré-natal recebido, verificou-se que, segundo o critério proposto por Takeda 10, $55 \%$ das mães adolescentes realizaram pré-natal considerado adequado contra $70 \%$ entre as mães de maior idade. Ao utilizar o critério proposto por Silveira et al. 11, a proporção de realização de pré-natal adequado foi de apenas 14\% entre as adolescentes e de $18 \%$ entre aquelas de maior idade.

Por fim, a Tabela 5 mostra que, ao se analisar a inadequação do pré-natal recebido ajustando para cor da pele, escolaridade, renda familiar per capita e estado civil, segundo Takeda 10, verificouse que a razão de prevalências entre adolescentes em relação às gestantes de maior idade caiu de $0,96(0,62-1,50)$ na análise bruta para $0,63(0,39$ 1,01) na análise ajustada. Queda semelhante foi observada em relação ao índice proposto por Silveira et al. 11 , caindo de 1,30 $(1,02-1,67)$ para $0,96(0,74-1,23)$. Isto sugere que as diferenças observadas em relação à inadequação do pré-natal não podem, neste caso, ser atribuídas às variáveis ajustadas no modelo.

\section{Discussão}

Este estudo mostrou que as mães adolescentes estiveram, de forma sistemática, em desvantagem em relação às demais mães tanto no que se 
Assistência à gestação para mães de recém-nascidos. Município de Rio Grande, Rio Grande do Sul, Brasil, 2007.

\begin{tabular}{|c|c|c|c|}
\hline Variável & Mães adolescentes & Mães não adolescentes & Valor de $p$ * \\
\hline $\begin{array}{l}\text { Realizaram pelo menos uma } \\
\text { consulta de pré-natal }\end{array}$ & $95,7 \%$ & $95,8 \%$ & 1 \\
\hline \multirow{2}{*}{\multicolumn{4}{|c|}{$\begin{array}{l}\text { Local de realização da maioria das } \\
\text { consultas de pré-natal }\end{array}$}} \\
\hline & & & \\
\hline Posto de saúde & $54,3 \%$ & $35,9 \%$ & $<0,001$ \\
\hline Ambulatório & $24,3 \%$ & $18,9 \%$ & \\
\hline Consultório particular/ & $20,2 \%$ & $43,8 \%$ & \\
\hline \multicolumn{4}{|l|}{ Convênio } \\
\hline Outro & $1,2 \%$ & $1,4 \%$ & \\
\hline \multicolumn{4}{|l|}{$\begin{array}{l}\text { Número de consultas de pré-natal } \\
\text { realizadas }\end{array}$} \\
\hline 6 ou mais & $61,1 \%$ & $74,8 \%$ & $<0,001$ \\
\hline 9 ou mais & $21,5 \%$ & $38,9 \%$ & $<0,001$ \\
\hline Mediana (intervalo interquartil) & $6(4-8)$ & $8(5-10)$ & $<0,001 \star \star \star$ \\
\hline \multicolumn{4}{|l|}{$\begin{array}{l}\text { Trimestre que iniciou as consultas } \\
\text { de pré-natal }\end{array}$} \\
\hline Primeiro & $58,5 \%$ & $77,4 \%$ & $<0,001$ \\
\hline Segundo & $37,7 \%$ & $20,5 \%$ & \\
\hline Terceiro & $3,9 \%$ & $2,1 \%$ & \\
\hline $\begin{array}{l}\text { Mediana de mês de início das } \\
\text { consultas (intervalo interquartil) }\end{array}$ & $3(2-4)$ & $2(1-3)$ & $<0,001 \star \star$ \\
\hline $\begin{array}{l}\text { Fizeram todo o pré-natal com o } \\
\text { mesmo profissional }\end{array}$ & $70,2 \%$ & $78,1 \%$ & $<0,001$ \\
\hline \multicolumn{4}{|l|}{ Exames laboratoriais realizados } \\
\hline \multicolumn{4}{|l|}{ no pré-natal } \\
\hline Sangue & $94,3 \%$ & $95,2 \%$ & 0,4 \\
\hline Urina & $93,3 \%$ & $94,4 \%$ & 0,3 \\
\hline HIV & $94,2 \%$ & $94,8 \%$ & 0,6 \\
\hline Sífilis & $90,8 \%$ & $92,8 \%$ & 0,2 \\
\hline Ultrassonografia pélvica & $88,8 \%$ & $93,2 \%$ & 0,001 \\
\hline Total & 516 & 2.041 & \\
\hline
\end{tabular}

* Teste de qui-quadrado de Fisher;

** Teste de qui-quadrado Pearson para comparação de valores acima da mediana.

refere a características socioeconômicas quanto na assistência recebida durante a gestação e o parto. As adolescentes possuíam pior nível de escolaridade e renda familiar, viviam mais comumente sem companheiro, realizaram um menor número de consultas de pré-natal, iniciaram estas consultas mais tardiamente, referiram com maior frequência a presença de anemia e corrimento vaginal patológico e foram mais submetidas ao uso de fórcipe e episiotomia.

O baixo percentual de perdas pode ser atribuído à facilidade de acesso as parturientes nas maternidades. As poucas mães não encontradas foram, na sua quase totalidade, oriundas de quartos particulares destes hospitais, portanto de melhor nível socioeconômico que as demais, o que supostamente aumentaria a diferença entre os grupos estudados. Estas mães não possuem um tempo mínimo de permanência no hospital. Além disso, algumas delas nem mesmo aparecem na lista diária de partos realizados, o que dificultou incluí-las no estudo.

Neste estudo, 20,2\% das mães riograndinas eram constituídos por adolescentes. Este percentual é praticamente o mesmo referido pelo Departamento de Informática do SUS (DATASUS) $(19,8 \%)$ para o município no mesmo período 13 . Isto evidencia a boa qualidade dos dados coletados 
Tabela 3

Assistência ao parto para mães de recém-nascidos. Município de Rio Grande, Rio Grande do Sul, Brasil, 2007.

\begin{tabular}{|c|c|c|c|}
\hline Variável & Mães adolescentes & $\begin{array}{l}\text { Mães não } \\
\text { adolescentes }\end{array}$ & Valor de $p^{*}$ \\
\hline Profissional que acompanhou no pré-parto & & & 0,04 \\
\hline Médico & $24,8 \%$ & $28,1 \%$ & \\
\hline Estudante & $55,6 \%$ & $55,6 \%$ & \\
\hline Enfermeira/Parteira & $19,6 \%$ & $15,7 \%$ & \\
\hline Ninguém & $0,0 \%$ & $0,6 \%$ & \\
\hline Teve o parto realizado por médico & $84,9 \%$ & $85,6 \%$ & 0,2 \\
\hline \multicolumn{4}{|l|}{ Tipo de parto } \\
\hline Normal & $59,9 \%$ & $45,5 \%$ & $<0,001$ \\
\hline Cesariana & $40,1 \%$ & $54,5 \%$ & \\
\hline Foi necessário usar fórceps & $10,7 \%$ & $6,2 \%$ & 0,01 \\
\hline Foram submetidas à episiotomia $(n=1.238)$ & $85,8 \%$ & $66,0 \%$ & $<0,001$ \\
\hline $\begin{array}{l}\text { Recém-nascido atendido por pediatra na sala } \\
\text { de parto }\end{array}$ & $90,8 \%$ & $94,1 \%$ & 0,04 \\
\hline \multicolumn{4}{|l|}{ Tipo de assistência } \\
\hline Pública (SUS) & $91,9 \%$ & $75,9 \%$ & $<0,001$ \\
\hline Particular & $1,7 \%$ & $5,7 \%$ & \\
\hline Convênio & $6,4 \%$ & $18,4 \%$ & \\
\hline Total & 516 & 2.041 & \\
\hline
\end{tabular}

* Teste de qui-quadrado de Fisher.

Tabela 4

Características demográficas, antropométricas e presença de asfixia entre os recém-nascidos. Município de Rio Grande, Rio Grande do Sul, Brasil, 2007.

\begin{tabular}{|c|c|c|c|}
\hline Variável & Mães adolescentes & Mães não adolescentes & Valor de $p$ * \\
\hline \multicolumn{4}{|l|}{ Sexo } \\
\hline Masculino & $49,3 \%$ & $51,7 \%$ & 0,3 \\
\hline Feminino & $50,6 \%$ & $48,3 \%$ & \\
\hline \multicolumn{4}{|l|}{ Peso ao nascer (g) } \\
\hline$<2.500$ & $7,1 \%$ & $7,9 \%$ & 0,2 \\
\hline $2.500-2.999$ & $24,0 \%$ & $22,5 \%$ & \\
\hline $3.000-3.499$ & $44,4 \%$ & $40,8 \%$ & \\
\hline$\geq 3.500$ & $24,6 \%$ & $28,8 \%$ & \\
\hline Média em gramas (DP) & $3.172(525)$ & $3.212(559)$ & $0,1 * *$ \\
\hline Média de comprimento ao nascer (DP) & $47,8(2,8)$ & $47,9(2,7)$ & 0,4 ** \\
\hline Média de perímetro cefálico (DP) & $34,3(2,3)$ & $34,5(2,0)$ & $0,1 * \star$ \\
\hline \multicolumn{4}{|l|}{ Idade gestacional (DUM $n=1.950)$} \\
\hline $\begin{array}{l}\text { Nasceram prematuros } \\
(<37 \text { semanas })\end{array}$ & $19,6 \%$ & $15,7 \%$ & 0,08 \\
\hline Média em semanas (DP) & $38,7(3,5)$ & $38,9(2,8)$ & 0,3 ** \\
\hline Nasceram vivos & $98,1 \%$ & $98,6 \%$ & 0,4 \\
\hline \multicolumn{4}{|l|}{ Índice de Apgar } \\
\hline$\geq 8$ no primeiro minuto & $79,8 \%$ & $83,1 \%$ & 0,09 \\
\hline$\geq 8$ no quinto minuto & $96,4 \%$ & $96,7 \%$ & 0,8 \\
\hline Total & 516 & 2.041 & \\
\hline
\end{tabular}

DP: desvio-padrão; DUM: data da última menstruação.

* Teste de qui-quadrado de Fisher;

** Teste-t. 


\section{Figura 1}

Adequação do atendimento pré-natal conforme critérios de Takeda 10 e de Silveira et al. ${ }^{11}$, estratificado por idade materna. Município de Rio Grande, Rio Grande do Sul, Brasil, 2007 ( $n=2.557)$.

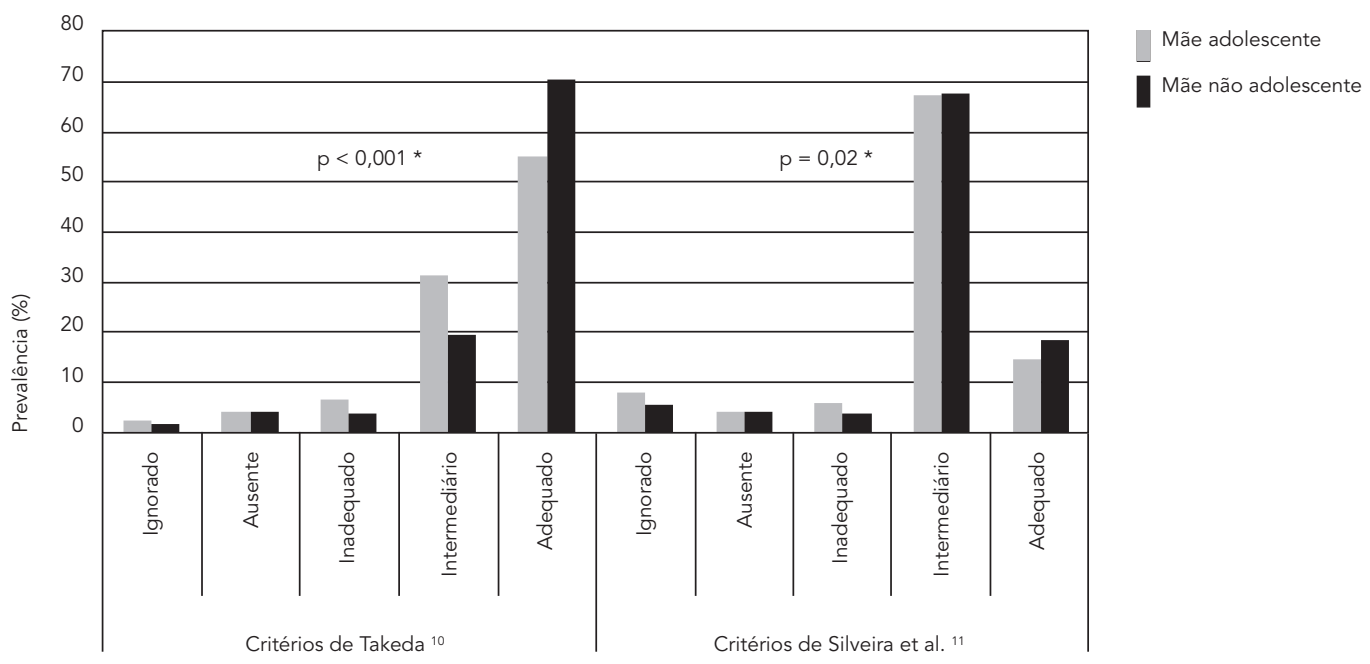

Adequação do atendimento pré-natal

* Teste de qui-quadrado de Fisher

\section{Tabela 5}

Análise bruta e ajustada da associação entre idade materna e pré-natal inadequado (segundo critérios de Takeda 10 ou de Silveira et al. 11 ). Município de Rio Grande, Rio Grande do Sul, Brasil, 2007.

\section{Variável}

Pré-natal inadequado *

Segundo Takeda 10

n $\%$

Bruta

RP (IC95\%)

$\mathrm{p}=0,9 * \star \star$
1,00

$2.006 \quad 4,7$

$0,96(0,62-1,5)$

RP (IC95\%)

$p=0,06$ ***

$\begin{array}{lll}\text { Ajustada ** } & \text { n } & \%\end{array}$

$\begin{array}{lll}1,00 & 1.925 & 11,3\end{array}$

Segundo Silveira et al. 11

Bruta Ajustada **

RP (IC95\%) RP (IC95\%)

IC95\%: intervalo de 95\% de confiança; RP: razão de prevalência.

* Pré-natal ausente foi considerado como inadequado nas análises;

** Ajustada para cor da pele autorreferida, escolaridade, renda familiar per capita e estado civil;

*** Teste de Wald de heterogeneidade. 
de rotina através do Sistema de Informações sobre Nascidos Vivos (SINASC). Esta taxa de gravidez na adolescência é igual ao observado para o Brasil em 2007 (21,1\%) e cerca de 15\% maior em relação à observada para o Rio Grande do Sul neste mesmo ano 13. É amplamente sabido que a gravidez na adolescência é mais comum entre aquelas de menor renda familiar e escolaridade 1,3. Estas mulheres iniciam vida sexual, engravidam e têm o parto mais cedo que as demais 4,6,14,15. A chamada metade sul do Rio Grande do Sul, região que inclui o Município de Rio Grande, é a mais pobre do estado. Isto pode, talvez, auxiliar na explicação de maior taxa em relação à observada para o Rio Grande do Sul como um todo.

Dezessete por cento de todas as mães incluídas neste estudo viviam sem companheiro. Este percentual foi 2,6 vezes maior entre mães adolescentes em relação às demais (35\% x 13\%).

Este é um achado muito frequente e reflete tão somente o desfecho de uma sucessão de equívocos, que se inicia com atividades sexuais em idade precoce, não utilização de método contraceptivo, gravidez, relações efêmeras com curto período de duração e separação ou, não raramente, o abandono por parte do namorado/ companheiro 3,16 .

Gravidez na adolescência é uma das três variáveis reprodutivas associadas à maior morbimortalidade materno-infantil 1. Comparadas às demais, filhos de mães com menos de 20 anos de idade apresentam risco cerca de $30 \%$ maior de morrer nos primeiros anos de vida. A presença de comorbidade no período gestacional como, por exemplo, anemia, hipertensão, infecção do trato urinário, corrimento vaginal patológico, é muito mais comum entre adolescentes do que em outras idades 1 . Todas estas co-morbidades, em maior ou menor grau, favorecem a ocorrência de prematuridade, principal determinante da morbimortalidade infantil no primeiro ano de vida ${ }^{17}$. No entanto, não foi isto que se observou neste estudo. A fim de elucidar isto para os dois grupos etários estudados, realizou-se análise ajustada por renda, tendo como desfecho estas comorbidades. Utilizando regressão de Poisson, verificou-se que as razões de prevalências mostraram maior probabilidade de ocorrência de co-morbidades entre as mulheres de maior idade em relação às adolescentes. Pelo menos neste estudo, ser adolescente não mostrou risco para este tipo de intercorrências durante a gravidez.

O uso de fórcipe entre todas as gestantes foi de $7 \%$, variando de $11 \%$ entre adolescentes e $6 \%$ em relação às demais gestantes. O uso de fórcipe indica parto complicado, o que espera ser mais comum entre as adolescentes, embora não se te- nha encontrado na busca realizada referências que dessem suporte de forma mais consistente a esta informação. Ao que parece, o uso de fórcipe ocorreu em demasia entre as parturientes estudadas.

A ocorrência de episiotomia entre todas as gestantes foi de $72 \%$, variando de $86 \%$ entre as adolescentes e $66 \%$ entre as demais mães, o que revela excesso de risco da ordem de $30 \%$. Este procedimento cirúrgico tem por objetivo facilitar o trabalho de parto e reduzir o risco de laceração perineal, mas não deve ser uma indicação de rotina 18. Claramente o uso deste recurso entre as mães de recém-nascidos de Rio Grande, sobretudo entre as adolescentes, mostrou-se abusivo, o que evidencia a ocorrência de intervenção médica desnecessária.

Setenta e nove por cento dos partos em Rio Grande foram realizados no sistema público de saúde. Entre as adolescentes, esta proporção foi de $92 \%$, ou, seja, $21 \%$ maior em relação àquelas de maior idade. Prevalência semelhante foi, também, verificada em outras localidades 13,19. A gratuidade da rede pública leva àqueles de menor recurso a procurar por ela, o que inclui a quase totalidade dos partos entre as adolescentes.

Independente do critério utilizado seja Takeda 10 ou Silveira et al. 11 a proporção de realização de pré-natal inadequado foi na comparação de proporções significativamente maior entre as adolescentes. Esta proporção que recebeu prénatal inadequado variou entre $25 \%$ utilizando-se o critério proposto por Silveira et al. ${ }^{11}$ e $30 \%$ para Takeda ${ }^{10}$. No entanto, ao se ajustar para nível sócio-econômico e características demográficas, esta diferença desapareceu. Isto sugere que as diferenças observadas não podem ser atribuídas às variáveis incluídas no modelo de ajuste.

Estes resultados permitem sugerir algumas intervenções capazes de melhorar indicadores de saúde materno-infantil no município, sobretudo entre gestantes adolescentes:

- Universalizar a assistência à gestação e ao parto e priorizar o seu atendimento nos serviços públicos de saúde - $4 \%$ das riograndinas não fizeram uma única consulta de pré-natal;

- Envidar esforços para que todas iniciem o prénatal no primeiro trimestre, que realizem pelo menos seis consultas e completem todos os exames clínicos e laboratoriais básicos;

- Incentivar a realização de pré-natal através de campanhas coletivas;

- Estimular a busca ativa pelos agentes comunitários de saúde de gestantes no domicilio;

- Capacitar os profissionais da rede básica de saúde a oferecer todos os exames clínicos necessários, sobretudo exame das mamas, citopatológico de colo uterino e ginecológico; 
- Aumentar a oferta de exames laboratoriais, particularmente VDRL;

- Ensinar sobre cada um dos procedimentos (clinico ou laboratorial) a que deveria ser submetida em cada consulta do pré-natal e cobrar por ele durante a consulta;

- Desencorajar a realização de episiotomia como procedimento de rotina, visto não haver evidência suficiente de efeito benéfico a todas àquelas que sofreram intervenção;

\section{Resumo}

Este estudo teve por objetivo comparar a assistência à gestação e ao parto entre mães adolescentes e não adolescentes residentes no Município de Rio Grande, Rio Grande do Sul, Brasil. Entre 1o de janeiro e 31 de dezembro de 2007 aplicou-se questionário padronizado a todas as mães destes recém-nascidos buscando informações sobre cuidados recebidos do inicio da gravidez até momento do parto. Utilizou-se teste do qui-quadrado para comparar proporções. Um quarto (516) dos recém-nascidos era filho de mães adolescentes. Em relação às demais mães, uma menor proporção de adolescentes completou seis ou mais consultas de pré-natal (61\% x 75\%), iniciou o pré-natal no primeiro trimestre de gravidez (58\% $x 77 \%$ ), recebeu vacina antitetânica ( $81 \% \times 85 \%)$ e fez todo o pré-natal com o mesmo profissional ( $70 \% \times 78 \%$ ); no entanto, foram mais comumente suplementadas com sulfato ferroso (66\% x 57\%), submetidas a fórcipe (11\% x 6\%), à episiotomia (86\% x 66\%), e atendidas no SUS (92\% $x$ 76\%). Estes dados mostram que a assistência recebida pelas mães adolescentes foi sistematicamente pior àquela recebida pelas demais mães.

Saúde Materno-Infantil; Cuidado Pré-Natal; Gravidez; Adolescentes
- Realizar investigação interna visando avaliar a real necessidade do uso de fórcipe;

- Fortalecer a rede pública de saúde visto que responde por 9 em cada 10 nascimentos entre as adolescentes; e

- Seguir monitorando a saúde perinatal no município a fim de definir intervenções com maior potencial de impacto.

\section{Colaboradores}

J. A. Cesar foi responsável pela definição do delineamento, treinamento de entrevistadores, supervisão da coleta de dados, análise dos dados e redação final do artigo. R. A. Mendoza-Sassi contribuiu com a definição do delineamento, análise de dados e redação final do artigo. D. A. Gonzalez-Chica, P. S. Mano e S. M. GoulartFilha auxiliaram na análise dos dados e na redação final do artigo.

\section{Agradecimentos}

À Fundação de Amparo à Pesquisa do Estado do Rio Grande do Sul (FAPERGS/PPSUS, processo 0700090) e à Pastoral da Criança (003/2007). 


\section{Referências}

1. Victora CG. Intervenções para reduzir a mortalidade infantil pré-escolar e materna no Brasil. Rev Bras Epidemiol 2001; 4:3-69.

2. Aragão VM, Silva AAM, Aragão LF, Barbieri MA, Bettiol H, Coimbra LC, et al. Risk factors for preterm births in São Luís, Maranhão, Brazil. Cad Saude Publica 2004; 20:57-63.

3. Ministério da Saúde. Pesquisa Nacional de Demografia e Saúde da Criança e da Mulher - PNDS 2006: dimensões do processo reprodutivo e da saúde da criança. Brasília: Ministério da Saúde/ Centro Brasileiro de Análise e Planejamento; 2009. (Série G. Estatística e Informação em Saúde).

4. Goldemberg P, Figueiredo MCT, Silva RS. Gravidez na adolescência, pré-natal e resultados perinatais em Montes Claros, Minas Gerais, Brasil. Cad Saúde Pública 2005; 21:1077-86.

5. Ribeiro ER, Guimarães AM, Bettiol H, Lima DD, Almeida ML, de Souza L, et al. Risk factors for inadequate prenatal care use in the metropolitan area of Aracaju, Northeast Brazil. BMC Pregnancy Childbirth 2009; 9:31.

6. Carvacho IE, Mello MB, Morais SS, Silva JLP. Fatores associados ao acesso anterior à gestação a serviços de saúde por adolescentes gestantes. Rev Saúde Pública 2008; 42:886-94.

7. Frota DAL, Marcopito LF. Amamentação entre mães adolescentes e não-adolescentes, Montes Claros, MG. Rev Saúde Pública 2004; 38:85-92.

8. Silva IS. Cancer epidemiology: principles and methods. Lyon: World Health Organization/International Agency for Research on Cancer; 1999.

9. Kessner DM. Screening high-risk populations: a challenge to primary medical care. J Community Health 1976; 1:216-25.

10. Takeda S. Avaliação da qualidade do pré-natal: Programa para Gestantes em Unidade de Atenção à Saúde [Dissertação de Mestrado]. Pelotas: Universidade Federal de Pelotas; 1993.
11. Silveira DS, Santos IS, Costa JSD. Atenção pré-natal na rede básica: uma avaliação da estrutura e do processo. Cad Saúde Pública 2001; 17:131-9.

12. Kirkwood BR, Sterne J. Essential medical statistics. Oxford: Blackwell; 2003.

13. Departamento de Informática do SUS. Informações sobre nascimentos no Brasil. Brasília: Ministério da Saúde; 2010.

14. Simões VM, da Silva AA, Bettiol H, Lamy-Filho F, Tonial SR, Mochel EG. Características da gravidez na adolescência em São Luís, Maranhão. Rev Saúde Pública 2003; 37:559-65.

17. Chalem E, Mitsuhiro SS, Ferri CP, Barros MCM, Guinsburg R, Laranjeira R. Gravidez na adolescência: perfil sócio-demográfico e comportamental de uma população da periferia de São Paulo, Brasil. Cad Saúde Pública 2007; 23:177-86.

18. Bassani DG, Surkan PJ, Olinto MT. Inadequate use of prenatal services among Brazilian women: the role of maternal characteristics. Int Perspect Sex Reprod Health 2009; 35:15-20.

19. Silveira MF, Victora CG, Barros AJD, Santos IS, Matijasevich A, Barros FC. Determinants of preterm birth: Pelotas, Rio Grande do Sul State, Brazil, 2004 birth cohort. Cad Saúde Pública 2010; 26:185-94.

20. Carroli G, Belizan J. Episiotomy for vaginal birth. Cochrane Database Syst Rev 2009; (1):CD000081.

21. Matijasevich A, Santos IS, Silveira FS, Domingues MR, Barros AJD, Marco LM, et al. Inequities in maternal and child health postnatal visits among public and private patients:2004 Pelotas cohort study. BMC Public Health 2009; 9:335.

Recebido em 15/Jul/2010

Versão final reapresentada em 22/Mar/2011

Aprovado em 05/Abr/2011 